Zavgorodnia N. G., Mykhailenko N. V. Biometric changes in highly myopic eyes before and after phacoemulsification with intraocular lense implantation. Journal of Education, Health and Sport. 2021;11(04): 131-143. eISSN 2391-8306. DOI http://dx.doi.org/10.12775/JEHS.2021.11.04.014

https://apcz.umk.pl/czasopisma/index.php/JEHS/article/view/JEHS.2021.11.04.014

https://zenodo.org/record/4883646

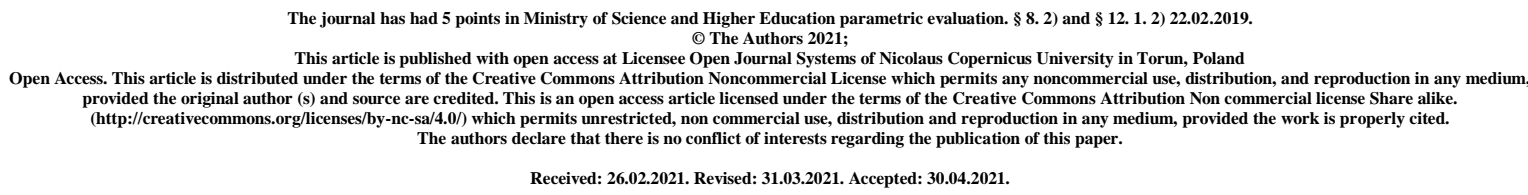

UDK: 617.753.2:[617.741-004.1-089.87+617.7-089.843-76]]-07:57.087.1

\title{
BIOMETRIC CHANGES IN HIGHLY MYOPIC EYES BEFORE AND AFTER PHACOEMULSIFICATION WITH INTRAOCULAR LENSE IMPLANTATION
}

\author{
N. G. Zavgorodnia ${ }^{1,2}$, N. V. Mykhailenko ${ }^{1,2}$ \\ ${ }^{1}$ Zaporizhzhia State Medical University, Department of Ophthalmology, Zaporizhzhya, \\ Ukraine \\ ${ }^{2}$ Clinic of Modern Ophthalmology «VISUS», Zaporizhzhya, Ukraine
}

N. G. Zavgorodnia 1,2 ORCID: https://orcid.org/0000-0002-5678-4196

N.V. Mykhailenko 1,2 ORCID: https://orcid.org/0000-0002-2779-8812

\section{Abstract}

High short-sightedness is a complicating factor in phacoemulsification cataract affecting the achievement of high visual results. There are no data in the literature that would reflect changes in the biometric parameters of eyes with high myopia after cataract phacoemulsification with intraocular lens implantation. The authors have shown the reliability of this surgical treatment, the absence of complications and a negative effect on the hydrodynamics of the eye. Patients underwent the ophthalmic examination and for patients preparing for phacoemulsification + IOL: A- and B-scanning, endothelial microscopy, optical biometry. In the group of patients with high myopia, regardless of the age, ranged from 3.1 $\mathrm{mm}$ to $3.9 \mathrm{~mm}$ with a mean of $3.52 \pm 0.34 \mathrm{~mm}$ (variation within $10 \%$ ), while in the group of patients with myopia and concomitant cataract the fluctuations in anterior chamber depth were recorded in the range from $2.6 \mathrm{~mm}$ to $4.4 \pm 0.1 \mathrm{~mm}$ with average values of $3.14 \pm 0.038$ 
$\mathrm{mm}$ (variation within 25\%). In the dynamics of treatment, a significant reduction in intraocular pressure according to Maklakov by $6.9 \%$ was noted in 6 months from baseline (p $<0.001$ ) - from $18.6 \pm 2.34$ to $17.4 \pm 1.09 \mathrm{~mm} \mathrm{Hg}$. Art. At the same time, it should be emphasized that the main effect of reducing intraocular pressure was expected already within 1 month $(17.9 \pm 1.06 \mathrm{~mm} \mathrm{Hg})$ after surgery $(3.9 \%, \mathrm{p}=0.006)$, followed by decrease by $3.1 \%$ after 6 months of follow-up $(\mathrm{p}=0.008)$. The research results showed a direct correlation between the endothelial layer density and the anterior chamber depth. Thus, before surgery $r=$ 0.248 , and after surgery $r=0.119$, i.e. after phacoemulsification with IOL implantation, the density of the endothelial layer depends on changes in the anterior chamber depth $(p<0.05)$. This research has shown that phacoemulsification with IOL implantation is accompanied by positive changes in the size of the mutually located structures of the anterior chamber of the eyeball.

\section{Key words: myopia; phacoemulsification; biometrics}

Introduction: Myopia or short-sightedness occurs in more than $50 \%$ of population in many developed countries and tends to increase. [1, 2]. Anomalies of refraction in high myopia significantly affect the quality of life of modern people as well as induce progression of other impairments. The morphological changes in eye balls and their separate anatomicoptical components in case of refractive disorder can be detected by means of various clinical and instrumental methods. Considering the pathological complications of myopia and other associated serious pathologies, short-sightedness is not only negatively affecting selfperception and work but is also one of the leading causes of blindness around the world [3].

Imbalance of intraocular and ocular volume parameters in the process of cataract genesis in myopic eyes may occur due to deterioration of ocular hemodynamics, changed conditions for the movement of aqueous humor, hydrodynamic disturbances, metabolic processes of the lens, changes in permeability of the lens capsule marked by swelling of the lens fibers [4].

Objective: to determine the biometric parameters and their effect in highly myopic eyes before and after phacoemulsification with IOL implantation and compare the obtained data with the data of the patients with high myopia without cataract.

Material and methods: the biometric parameters before and after phacoemulsification with IOL implantation (group I) were analyzed in 58 patients (96 eyes) with high myopia aged 29-78 years (mean age 55,9 $\pm 1,8$ ). Among them, there were 32 women 
$(55,1 \%)$ and 26 men $(44,9 \%)$. The obtained data were compared with the group of patients with high myopia without cataract (group II).

It included 20 patients (33 eyes) aged 20-56 years (mean age 33,6 $\pm 2,2$ ), among them 12 women $(60 \%)$ and 8 men (40\%). It is difficult to make these groups comparable in terms of age because the patients with high myopia are more prone to cataract in young age than patients with other types of refractive pathology.

In the preoperative period all patients underwent the ophthalmic examination (autorefractometry, visometry, tonometry, biomicroscopy, perimetry, Amsler test, phosphene test, direct ophthalmoscopy, retina exam by Goldmann lens (where possible) and for patients preparing for phacoemulsification $+\mathrm{IOL}-(\mathrm{A}$ - and B-scanning ultrasonography using the UltraScan (Alcon, USA)), endothelial microscopy (endothelial biomicroscope SP-3000P (Topcon, Japan), optical biometry using the IOLMaster ${ }^{\circledR} 700$ (Carl Zeiss, Germany). Haigis and SRK-T formulas were used to calculate the optical power of IOL using the IOLMaster®700 (Carl Zeiss, Germany).

The patients were implanted with 59 monofocal intraocular lenses, the majority of which were the following: CT LUCIA 601P (Carl Zeiss, Germany), CT ASPHINA 409MV (Carl Zeiss, Germany) and NASPRO BBY (Appasamy Associates, India). Apart from this, 37 multifocal lenses were implanted, whereas the following lenses were prevailing: AT Lisa tri 839 (Carl Zeiss, Germany), MP Lisa 809M (Carl Zeiss, Germany) and MULTIDIFF 605 (Appasamy Associates, India).

All tests and surgical interventions were performed at the clinic of modern ophthalmology "VISUS". This clinic is the clinical site of the Department of Ophthalmology of Zaporizhzhia State Medical University. The patients were examined before and 1 and 6 months after the surgery. All the patients participating in the study granted their consent to the use of their data in the research paper.

The results were statistically processed using the programs Statistica 10, BiostatPro 6 and Microsoft Excel 2016. The normality of calculations and values in the samples was checked using the Shapiro-Wilk test. The arithmetic mean of the variation series (M) and its standard error $(\mathrm{m})$ were obtained to evaluate the quantitative data. Student's t-test was used for comparison of quantitative values in paired rows. The difference was considered significant at the values $\mathrm{p}<0,05$.

Results. It was found that in myopia there was an increase in anterior-posterior length of the eyeball and excessive convexity of the cornea. Thus, the anterior-posterior length of the eyeball in patients with high myopia was in the range from $25.63 \mathrm{~mm}$ to $30.8 \mathrm{~mm}$ and in 
average amounted to $27.79 \pm 0.34 \mathrm{~mm}$ (table 1) (with reference values of 22-24 mm). Obviously, it is the increase in the length of the eyeball by $3 \mathrm{~mm}$ or more that threatens a significant thinning of the retinal and vascular membranes of the eye, resulting in significantly reduced visual acuity.

At the same time, the anterior-posterior length of the eyeball in patients with high myopia complicated by lens opacity ranged from $25.9 \mathrm{~mm}$ to $33.1 \mathrm{~mm}$ and averaged $28.39 \pm$ 0.27 , which was not statistically significantly different from the values in the group of patients with isolated myopia $(\mathrm{P}<0.05)$ (table 1$)$.

It is known [5] that changes in the biomechanical (supporting) properties of the scleral capsule of the eye induce the progression of myopia with developing complications. The more so, stretching at myopization, first of all, concerns the oraequatorial divisions of the sclera, and only then the posterior pole of an eye is involved in this process.

Table 1

Optical biometrics in the eyes with high myopia before and after phacoemulsification with intraocular lens implantation, $\mathrm{M} \pm \mathrm{m}$

\begin{tabular}{|c|c|c|c|c|}
\hline Parameter & Myopia without & \multicolumn{3}{|c|}{ Myopia with cataract (n=96) } \\
\cline { 3 - 5 } & $\begin{array}{c}\text { cataract } \\
(\mathrm{n}=33)\end{array}$ & $\begin{array}{c}\text { Before surgery } \\
(\mathrm{PHACO}+\mathrm{IOL})\end{array}$ & $\begin{array}{c}1 \text { month after } \\
\text { surgery }\end{array}$ & $\begin{array}{c}6 \text { months after } \\
\text { surgery }\end{array}$ \\
\hline $\begin{array}{c}\text { Anterior-posterior } \\
\text { length of the eye (mm) }\end{array}$ & $27,89 \pm 0,34$ & $28,39 \pm 0,27$ & $28,36 \pm 0,27$ & $28,36 \pm 0,27$ \\
\hline $\begin{array}{c}\text { Anterior chamber depth } \\
(\mathrm{mm})\end{array}$ & $3,52 \pm 0,34$ & $3,14 \pm 0,03^{*}$ & $4,98 \pm 0,05^{*}, \#$ & $4,98 \pm 0,05^{*}, \#$ \\
\hline $\begin{array}{c}\text { Anterior chamber depth } \\
\text { / anterior-posterior } \\
\text { length }\end{array}$ & $11,54 \pm 0,1$ & $12,46 \pm 0,11^{*}$ & $17,55 \pm 0,18^{*}, \#$ & $17,55 \pm 0,18^{*}, \#$ \\
\hline Size of lens (mm) & $3,834 \pm 0,1$ & $4,378 \pm 0,036^{*}$ & $1,0 \pm 0,1^{*}, \#$ & $1,0 \pm 0,1^{*}, \#$ \\
\hline
\end{tabular}

Note: * - statistically significant difference compared to the parameters in patients with myopia without cataract, $\mathrm{P}<0,05$; \# - statistically significant difference compared to the parameters in patients before $\mathrm{PHACO}+\mathrm{IOL}, \mathrm{P}<0,05$.

Changes in the anterior-posterior length of the eye can lead to disruption of the normal morphology of the eyeball, which can affect the condition of the tissues in the anterior chamber of the eye, especially before and after cataract surgery.

The average value of the anterior chamber depth of the eye in the group of patients with high myopia, regardless of the age, ranged from $3.1 \mathrm{~mm}$ to $3.9 \mathrm{~mm}$ with a mean of 3.52 $\pm 0.34 \mathrm{~mm}$ (variation within $10 \%$ ), while in the group of patients with myopia and concomitant cataract the fluctuations in anterior chamber depth were recorded in the range 
from $2.6 \mathrm{~mm}$ to $4.4 \pm 0.1 \mathrm{~mm}$ with average values of $3.14 \pm 0.038 \mathrm{~mm}$ (variation within $25 \%$ ), i.e. individual variability of anterior chamber depth among people with myopia and myopia complicated by cataract is registered 2.5 times more often. At the same time, there was a significant difference in mean values of the anterior chamber depth in the groups with high myopia with and without clouding of the lens $(\mathrm{P}>0.05)$ (table 1).

In addition, patients with high myopia and concomitant cataract had an increase in the size of the lens, which was $14 \%$ higher than the average lens size in patients with myopia, but without cataract (table 1). The increase in the size of the lens is apparently associated with the cataractogenic swelling of its fibers. Increase in the lens thickness may cause the iridocrystalline diaphragm to shift forward.

The results showed that one month after the surgery, the changes in biometric parameters were mainly reflected in the change of the anterior chamber depth. Thus, the anterior chamber was deeper than before the surgery with the established mean values of 4.98 $\pm 0.055 \mathrm{~mm}(\mathrm{P}<0.05)($ table 1$)$.

These changes in the anterior chamber of the eye may reflect the correction of hydrodynamic conditions in the eyes, in particular, intraocular pressure reduction.

In case of removal of the patient's own lens, the iris deviates to the posterior pole, the feature of its shape, which corresponded to the anterior surface of the lens, disappears, the distance from the corneal endothelium in the plane of the pupil increases.

Changes in the anterior-posterior length one month after the surgery were statistically insignificant compared to the parameters which had been observed in this group of patients before the surgery $(\mathrm{P}>0,05)$ (table 1$)$.

The positive changes that were observed in the patients during the first month after the surgery, were also observed 6 months later since there was no statistical difference in the measurements $(\mathrm{P}>0,05)$ (table 1).

As for the proposed coefficients of the ratio of intraocular structures anterior chamber depth/anterior-posterior length, as shown in the table, they differ even at equal values of the anterior-posterior length, but this is influenced by different depths of the anterior chamber due to increase of the lens during clouding.

The correlation analysis assessed the strength and nature of the relationship between the studied biometric parameters in the patients before surgery and the visual acuity values. We noted an inverse correlation between the values of the corrected visual acuity and the anterior-posterior length of the eye in the patients of all study groups (Fig. 1). The obtained correlation coefficients are statistically significant $(\mathrm{P}<0,05)$. 
Thus, if in the patients with high myopia there was a moderate inverse correlation with the value $r=-0.361$ (Fig. 1A), then in the patients with high myopia and cataract there was a stronger dependency of corrected visual acuity on the anterior-posterior length of the eye with the correlation value $r=-0.838$ (Fig. 1B). After phacoemulsification with IOL implantation, the corrected visual acuity depended to a lesser extent on the anterior-posterior length of the eye, as the value of $r$ decreased to -0.380 one month after surgery and was fixed at this level 6 months after surgery $(\mathrm{P}<0.05)$ (Fig. 1B).

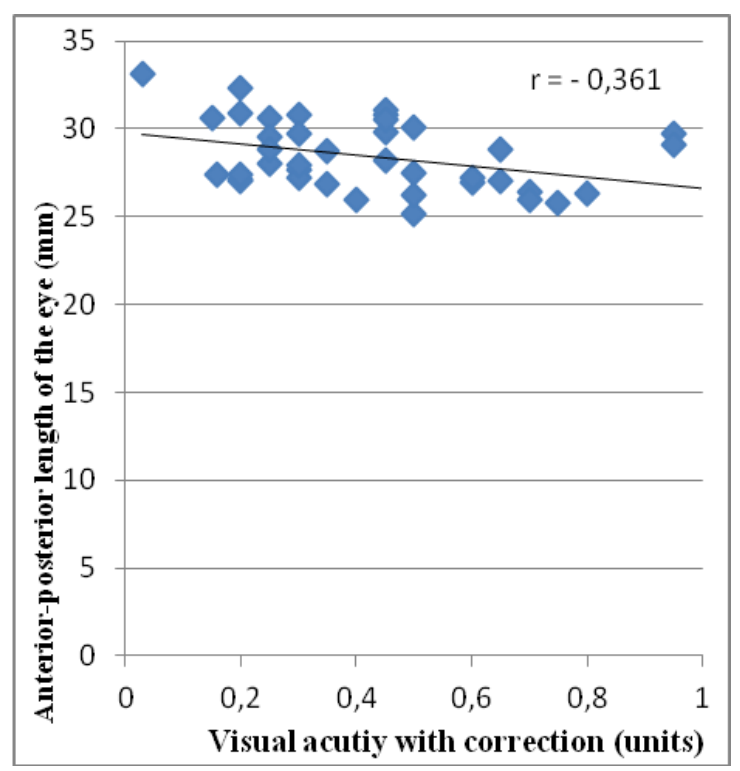

A

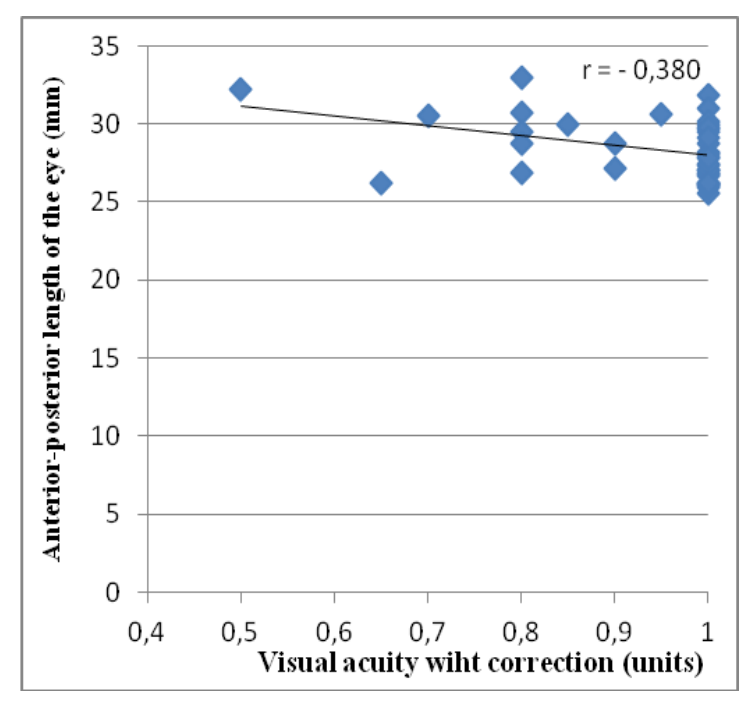

C

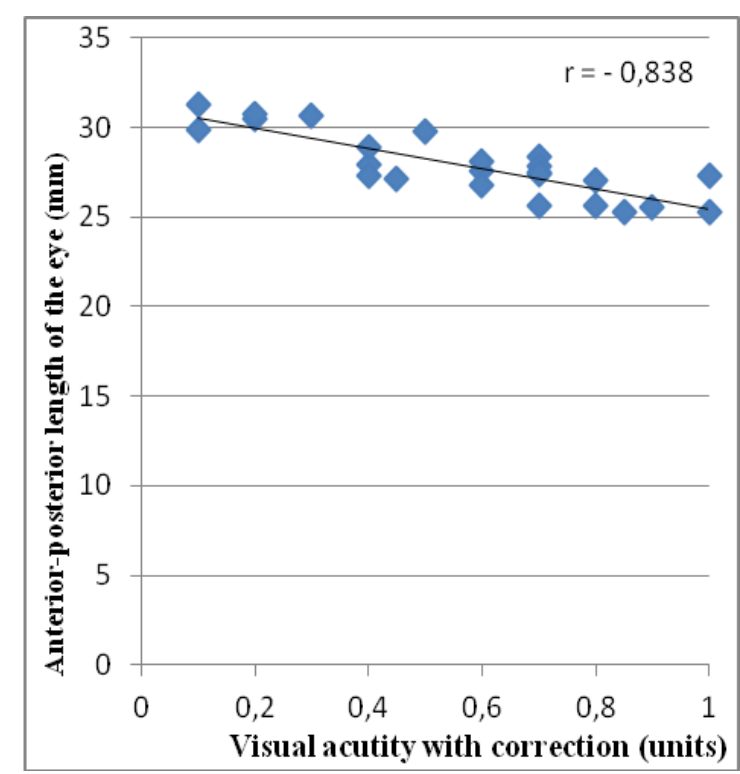

B

Fig 1. Correlative dependency between the anterior-posterior length of the eye and visual acuity in patients with high myopia complicated by cataract before and after surgery

Note: A - group of patients with high myopia; B - patients with cataract and high myopia before surgery; $\mathrm{C}$ - patients 6 months after phacoemulsification with IOL implantation. 
Thus, with increasing anterior-posterior length of the eye, corrected visual acuity decreases in patients with high myopia complicated by lens opacity. After phacoemulsification with IOL implantation, this dependency decreases.

Along with changes in the anterior-posterior length of the eye, visual acuity may be affected by changes in the anterior chamber depth. The analysis of the results showed that in highly myopic eyes there is an average direct correlation between visual acuity and anterior chamber depth $(\mathrm{r}=0.395)$ (Fig. 2A).

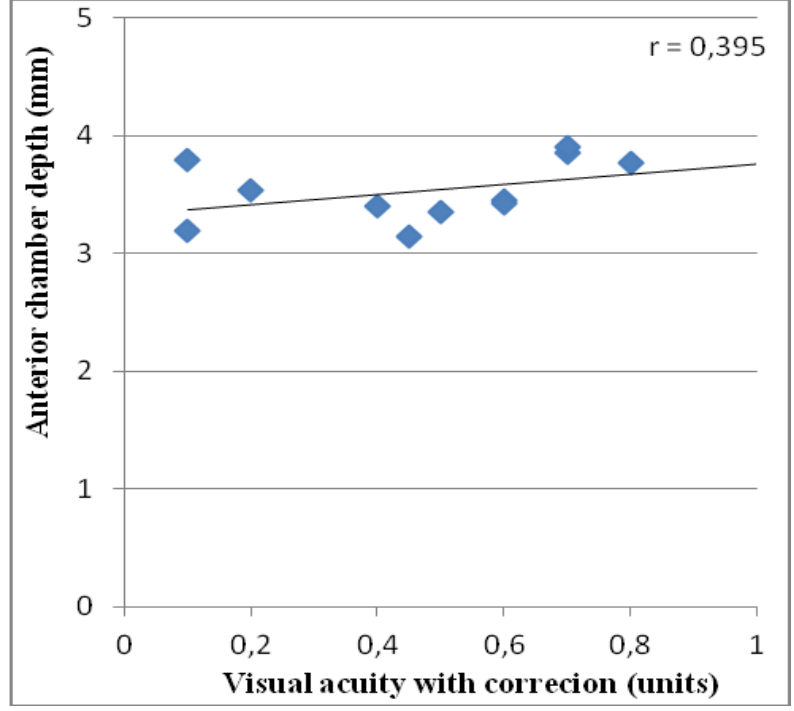

A

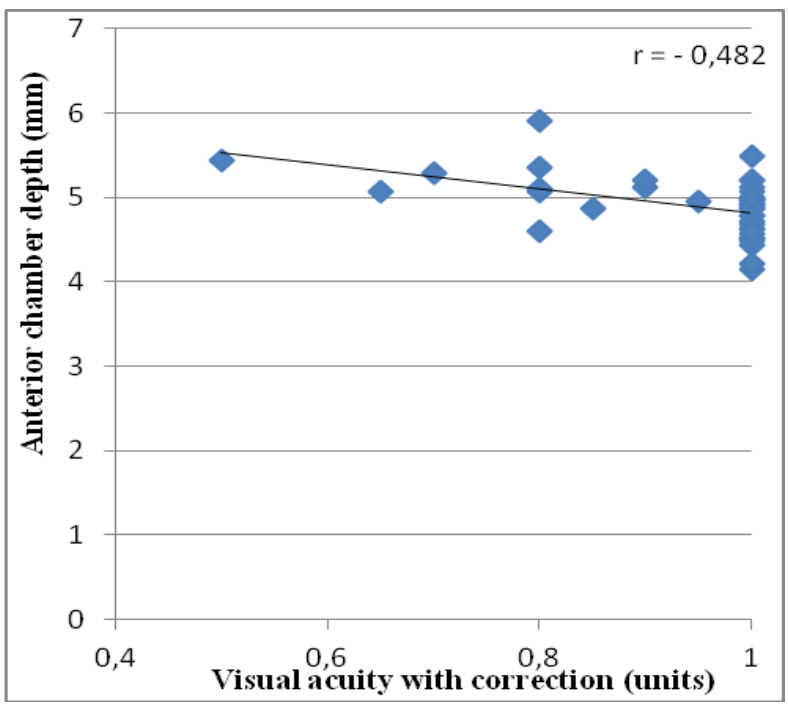

$\mathrm{C}$

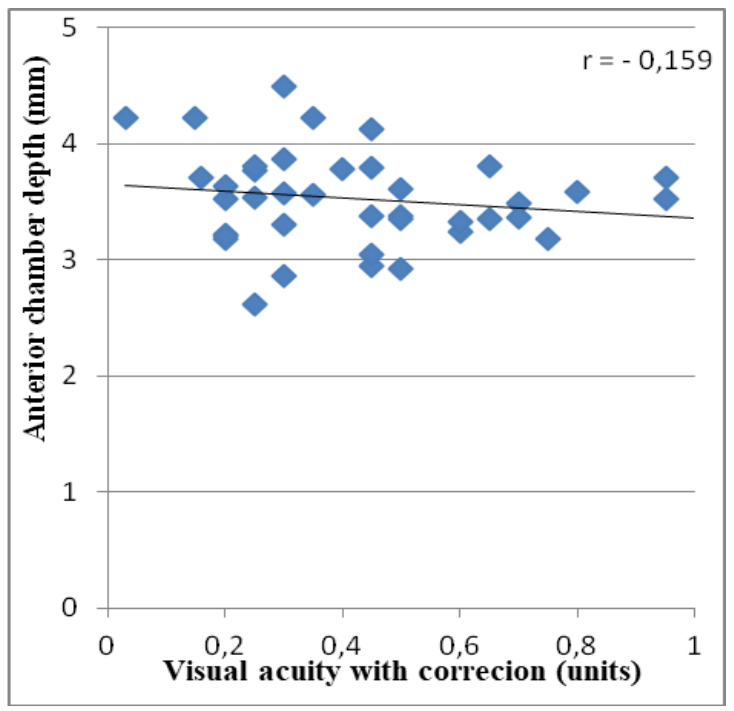

B

Fig. 2. Correlative dependency between anterior chamber depth and visual acuity in patients with high myopia complicated by cataract before and after surgery

Note: A - group of patients with high myopia; B - patients with cataract and high myopia before surgery; $\mathrm{C}$ - patients 6 months after phacoemulsification with IOL implantation. 
At the same time, patients with high myopia and cataract have a weak inverse correlation between the anterior chamber depth and visual acuity $(r=-0.159)$ (Fig. 2B).

6 months after phacoemulsification with IOL implantation, there is an inverse correlation between the anterior chamber depth and visual acuity $(r=-0.482)$ (Fig. 2B). The explanation for this fact is that as the eye is stretched during high myopia, two significant changes occur. Firstly, a significant weakening of the refractive power as a whole due to increased radius of curvature of the anterior surface of the cornea, and, accordingly, weakening of its refractive power. Secondly, due to weakening of the refractive power of the lens [4].

Regarding the size of the lens in patients with high myopia, in case of isolated myopia a slight inverse correlation is observed $-\mathrm{r}=-0.122$ (Fig. 3A), and in patients with complicated cataract visual acuity does not depend on the size of the lens $(r=0,0006)$ (Fig. $3 B)$.

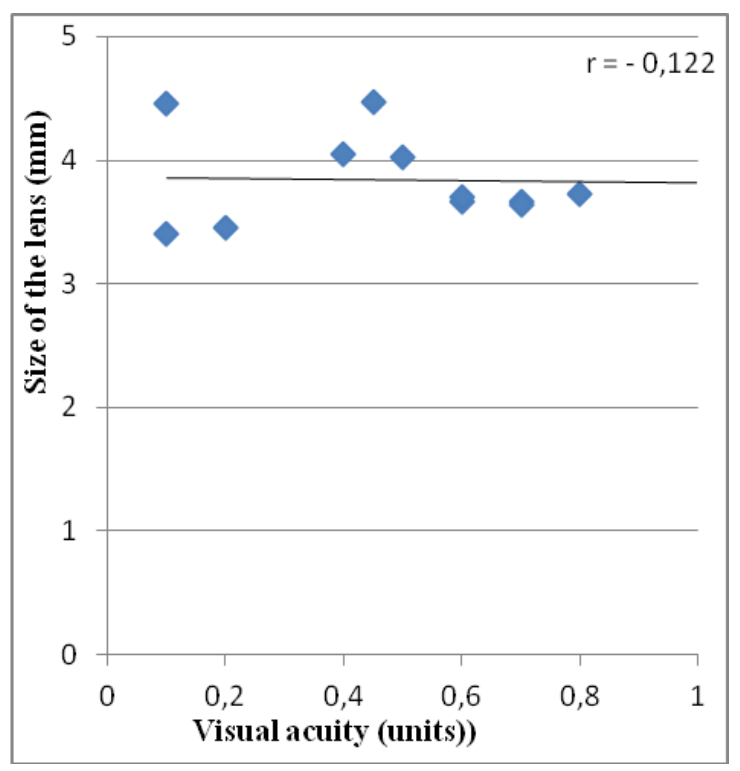

A

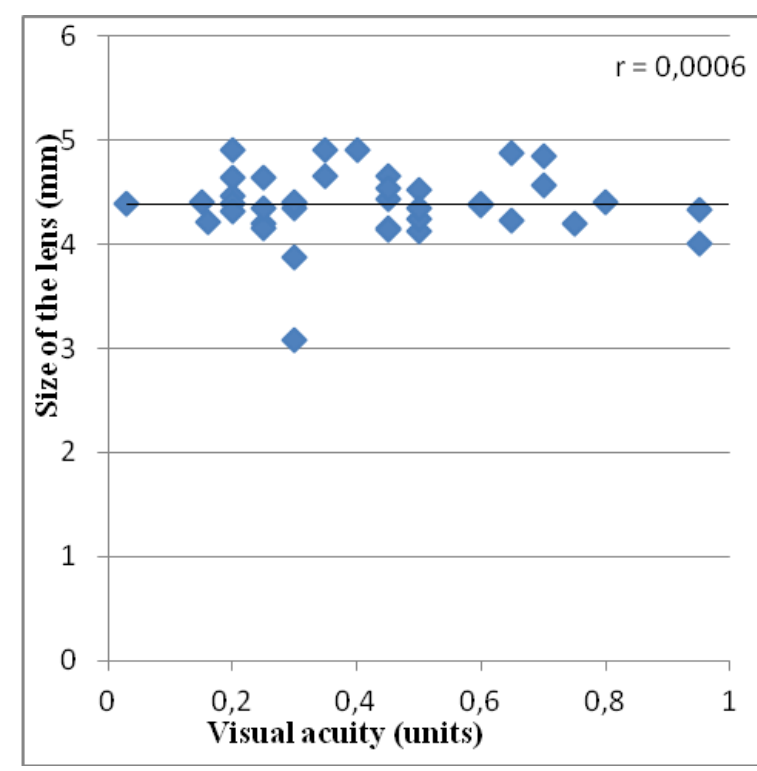

B

Fig. 3. Correlative dependency between the size of the lens and visual acuity in patients with high myopia complicated by cataract before surgery

Note: A - group of patients with high myopia; B - patients with cataract and high myopia before surgery.

Obviously, with the development of cataract in myopic eyes, visual acuity depends more on the intensity of the processes of clouding of the lens, rather than on its size.

The results of the study showed that uncomplicated phacoemulsification with IOL implantation expands the functional space of the structures of the anterior segment of the eye by deepening the anterior chamber, which means that the angle of the anterior chamber 
changes - it expands. Thus, the hydrodynamic parameters of the eye improve, namely the reduction of intraocular pressure (Fig. 4.) Therefore, after the surgery improvement is observed not only in visual functions, but also in the hydrodynamics of the eye, which contributes to the rehabilitation of this category of patients.

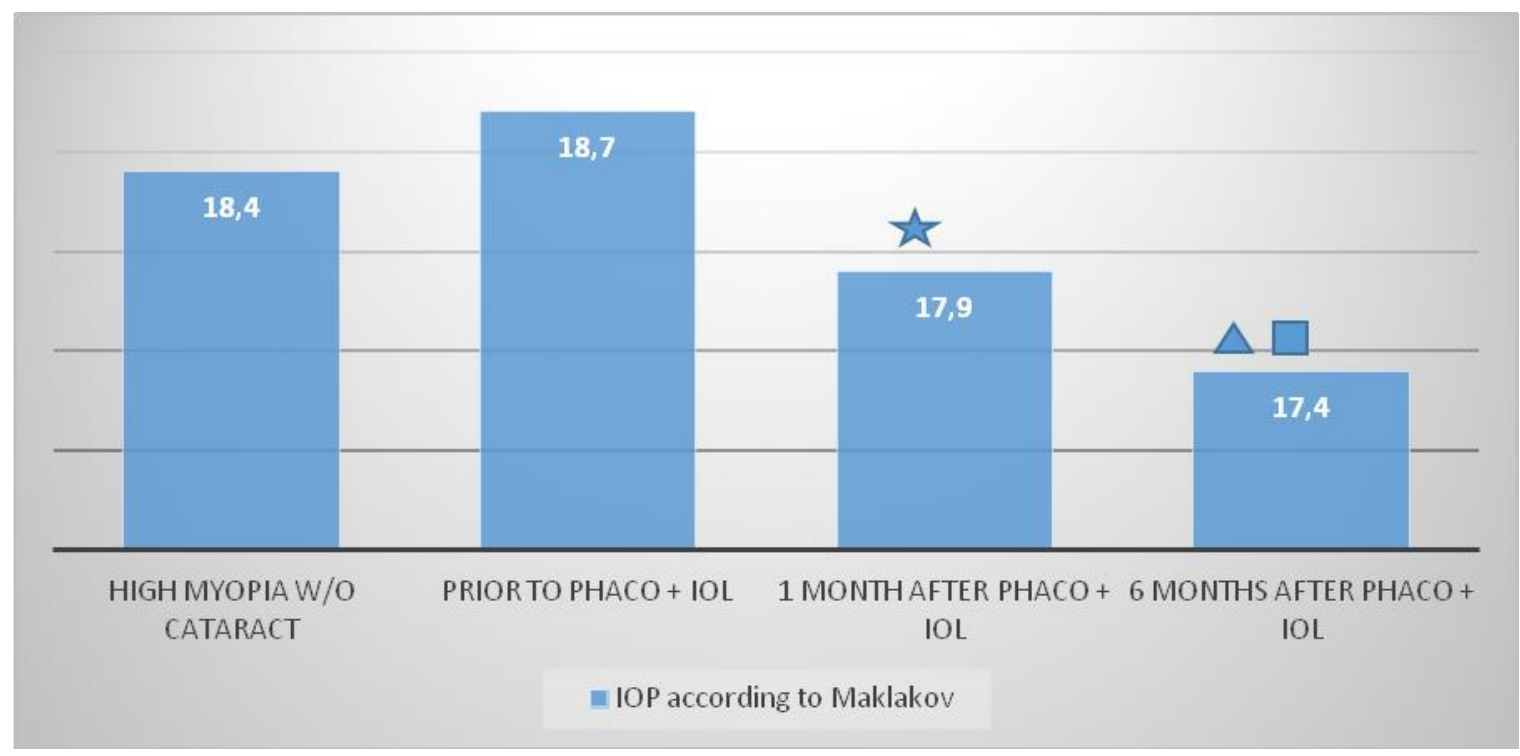

Fig. 4. IOP dynamics before and after PHACO + IOL, 1 month and 6 months after.

Notes $^{\wedge} \star-\mathrm{H}<0,05$ between the parameters before and after Phaco + IOL

$\Delta-\mathrm{P} 1<0,05$ between the parameters before and 6 months after Phaco + IOL

$\square-\mathrm{P} 2<0,05$ between the parameters 1 month and 6 months after Phaco + IOL

In the dynamics of treatment, a significant reduction in intraocular pressure according to Maklakov by $6.9 \%$ was noted in 6 months from baseline ( $\mathrm{p}<0.001)$ - from $18.6 \pm 2.34$ to $17.4 \pm 1.09 \mathrm{~mm} \mathrm{Hg}$. Art. At the same time, it should be emphasized that the main effect of reducing intraocular pressure was expected already within 1 month $(17.9 \pm 1.06 \mathrm{~mm} \mathrm{Hg})$ after surgery $(3.9 \%, p=0.006)$, followed by decrease by $3.1 \%$ after 6 months of follow-up $(p=$ $0.008)$.

In case of development of high myopia there can be a decrease in density of the cells of the endothelial layer. These features can affect the postoperative condition of the cornea [6]. In addition, endothelial cells may reflect the degree of surgical trauma.

The results showed that before the surgery the number of endothelial cells was 2577.3 \pm 55.01 cells $/ \mathrm{mm} 2$. At the same time, after PHACO + IOL there was a loss of endothelial cells by an average of about $10 \%$ with an average of $2313.5 \pm 74.24$ cells / mm2 (Fig. 5), which indicated a high positive effect of the surgery. Thus, according to researchers $[7,8]$, the 
complication of the surgery is evidenced by the loss of cells by $40 \%$, while the loss of endothelial cells by $10-19 \%$ indicates a positive result.

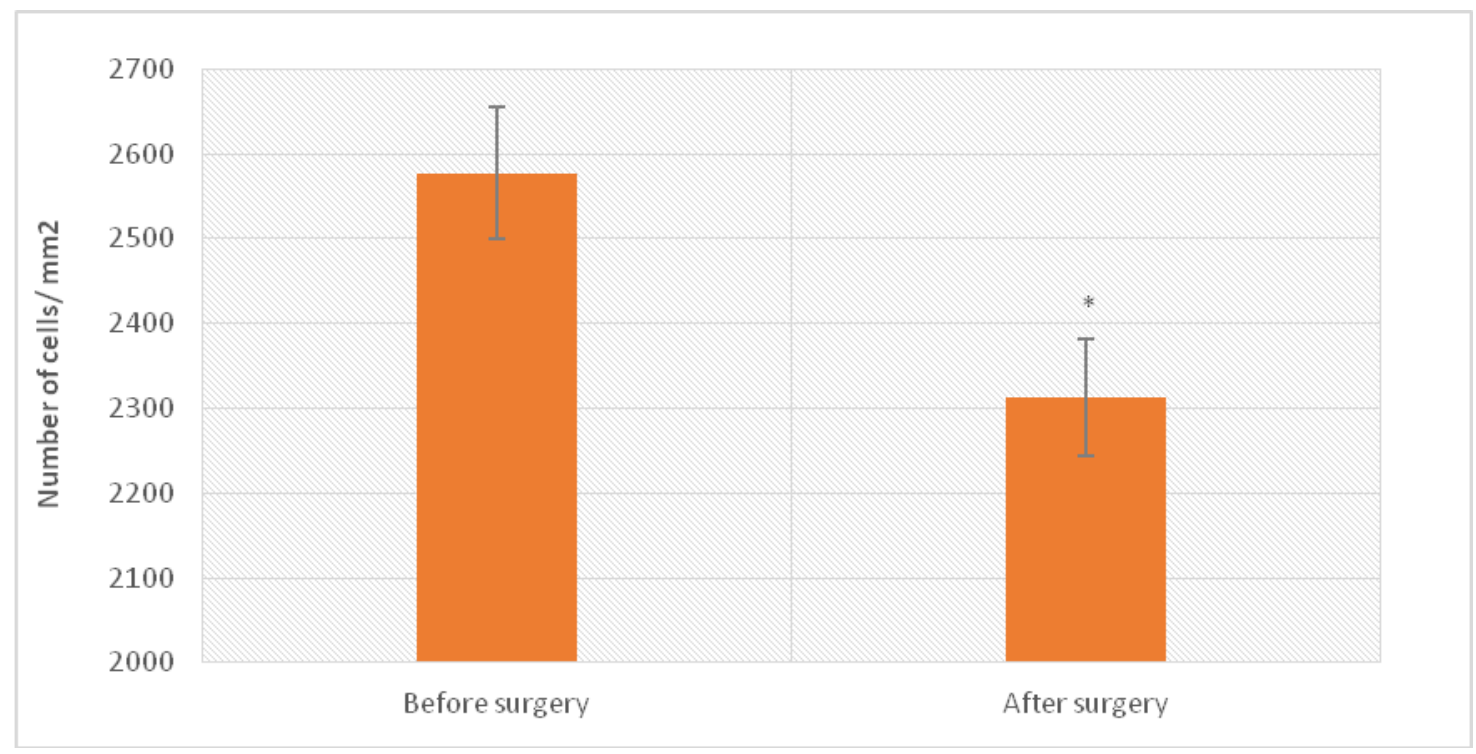

Fig. 5. Endothelial cell level in myopic eyes before and after phacoemulsification with intraocular lens implantation

Note: $*$ - statistically significant difference compared to the parameters in patients before PHACO + IOL, P $<0,05$.

Our correlation between anterior-posterior length and the number of endothelial cells in myopic eyes did not show a significant dependency between these parameters both before $(r=-0.003)$ and after $(r=0.003)$ phacoemulsification with IOL implantation (Fig. 6).

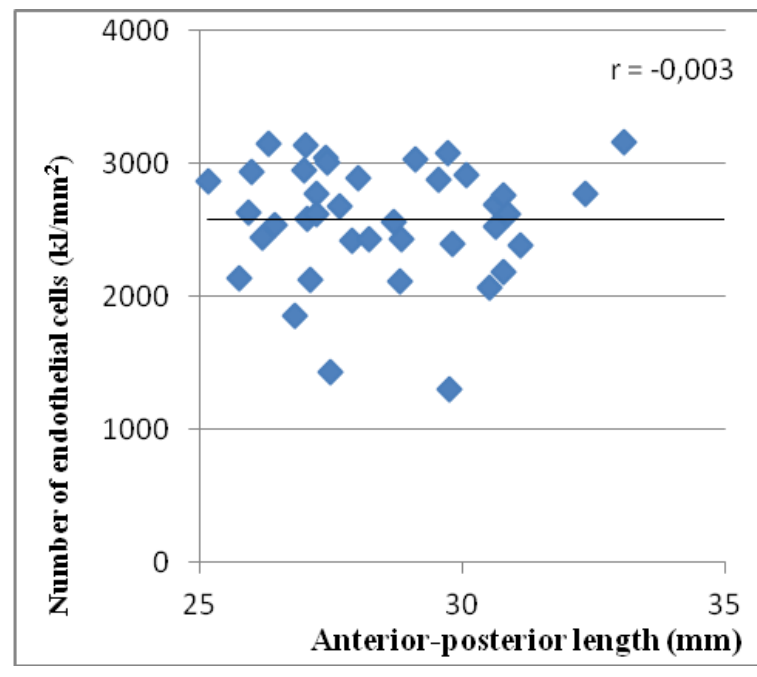

A

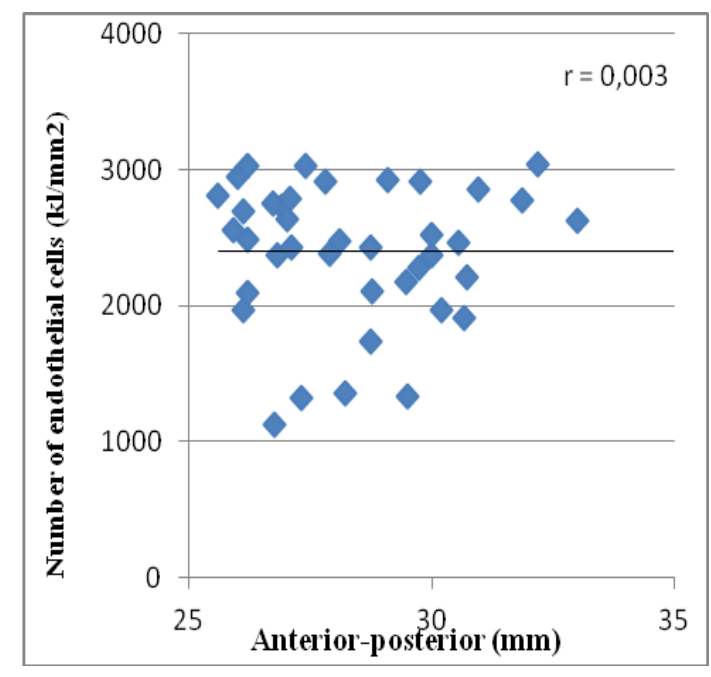

B

Fig. 6. Correlative dependency between anterior-posterior length and endothelial cell density in myopia before (A) and after (B) surgery 
At the same time, the research results showed a direct correlation between the endothelial layer density and the anterior chamber depth. Thus, before surgery $r=0.248$, and after surgery $r=0.119$, i.e. after phacoemulsification with IOL implantation, the density of the endothelial layer depends on changes in the anterior chamber depth ( $p$ <0.05) (Fig. 7). However, if we analyze these values in detail, the endothelial layer is more affected by phacoemulsification, during which ultrasound affects endothelial cells and changes their number, and therefore density.

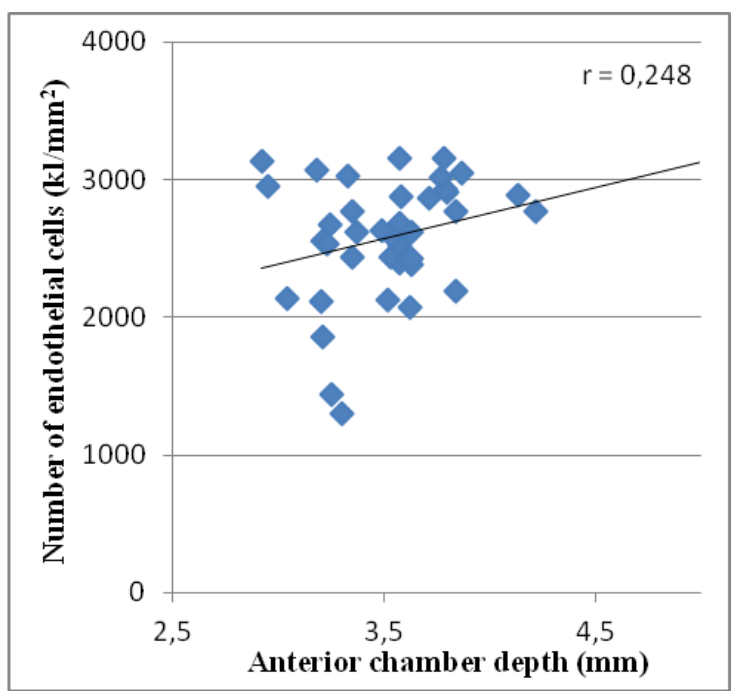

A

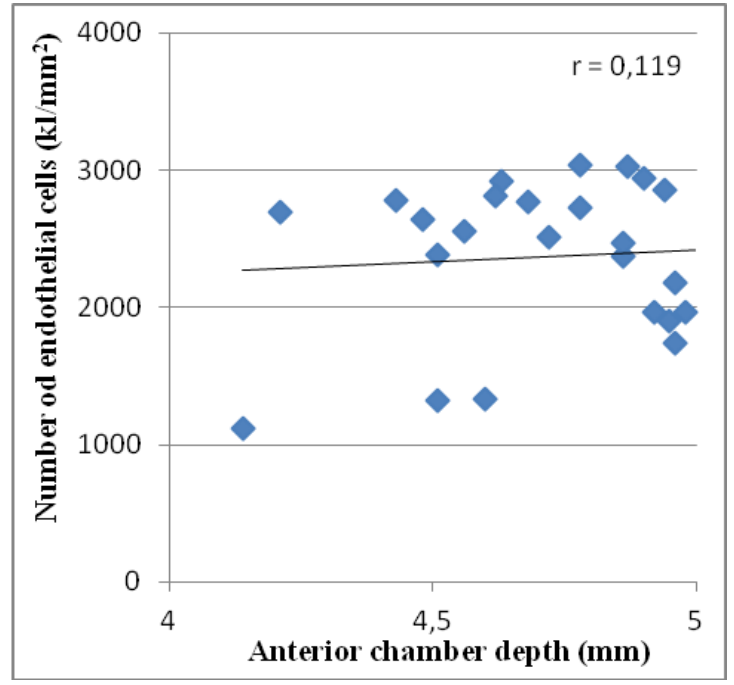

B

Fig. 7. Correlative dependency between anterior chamber depth and endothelial cell density in myopic eyes before (A) and after (B) surgery

Discussion: The results of the study showed a positive effect of phacoemulsification with implantation of an artificial lens on biometric changes of the eye in high myopia. Removal of the native lens and its replacement by a thin IOL cause shift of the iris root backward and modification of the angle profile of the anterior chamber. This increases the anterior chamber depth. The study helped to identify the correlative dependency between visual acuity and biometric parameters. Capsule sac fibrosis - ligament tension - traction of the ciliary body - decreased production of aqueous humor - these are the factors that lead to the hypotensive effect of phacoemulsification in the eyes with high myopia.

Elevated levels of IOP can affect the load on the scleral membrane of the eyeball and, along with structural, biochemical and biomechanical changes of the sclera, can contribute to its stretching in both sagittal and frontal directions [9]. Therefore, it is advisable to consider the refractive lens surgery before the appearance of cataract on the eyes with high myopia, or 
in the initial stage of cataract, and thus reduce the negative effects of myopia and cataract in general.

\section{Conclusion:}

1. This research has shown that phacoemulsification with IOL implantation is accompanied by positive changes in the size of the mutually located structures of the anterior chamber of the eyeball.

2. Increase in the depth of the anterior chamber and opening of its angle after PHACO + IOL in the eyes with high myopia improves the outflow of aqueous humor and reduces IOP after surgery.

3. It is advisable to perform phacoemulsification with IOL implantation in the early stages of cataract development in high myopia, in order to prevent transient increase in IOP and possible changes in the nerve fibers of the optic nerve.

\section{References:}

1 Holden B.A., Fricke T.R., Wilson D.A., Jong M., Naidoo K.S., Sankaridurg P., Wong T.Y., Naduvilath T.J., Resnikoff S. Global prevalence of myopia and high myopia and temporal trends from 2000 through 2050. Ophthalmology. 2016;123(5):1036-42.

2 PTVM de J. Myopia: its historical contexts. Br J Ophthalmol. 2018; 102(8): 1021-1027.

3 Holden B., Sankaridurg P., Smith E. et al. Myopia, an underrated global challenge to vision: Where the current data takes us on myopia control. Eye (Lond). 2014;28:142-146.

4 Niu L., Miao H., Han T., Ding L., Wang X., Zhou X. Visual outcomes of Visian ICL implantation for hi myopia in patients with shallow anterior chamber depth. BMC Ophthalmol. 2019;19(1):121.

5 Nguyen H.T.T., Nguyen D.T.T., Pham D.N., Tran A.P., Quyet D., Thai T.V., Nga V.T., Bac N.D. Ocular biometrics of vietnamese young adults with myopia. Open Access Maced J Med Sci. 2019;7(24):4283-4286

6 Lyu T., Wang L., Zhou L., Qin J., Ma H., Shi M. Regimen Study of High Myopia-Partial Reduction Orthokeratology. Eye Contact Lens. 2020; 46(3):141-146.

7 Reuschel A., Bogatsch H., Barth T., Wiedemann R. Comparison of endothelial changes and power settings between torsional and longitudinal phacoemulsification. $J$. Cataract. Refract Surg. 2010; 36:1855 -1861. 
8 Walkow T., Anders N., Klebe S. Endothelial cell loss after phacoemulsification: relation to preoperative and intraoperative parameters. J. Cataract Refract. Surg. 2000.26:727-732.

9 Lee K., Yang H., Kim J.Y., Seong G.J., Kim C.Y., Bae H.W. risk factors associated with structural progression in normal-tension glaucoma: intraocularupressure, systemic bloodupressure, and myopia. Invest Ophthalmol Vis Sci. 2020;61(8):35. 